\title{
Produção do híbrido “cachadia” em diferentes densidades de estocagem em sistema de recirculação de água
}

[Production of cachadia hybrid at different densities in a recirculating aquaculture system]

\author{
P.M.C. Faria ${ }^{1}$, R.K. $\mathrm{Luz}^{2}$, S.A. Prado ${ }^{2}$, E.M. Turra ${ }^{2}$, T.B.F. Jorge ${ }^{2}$, \\ A.M.Q. Lana ${ }^{2}$, E.A. Teixeira ${ }^{2 *}$ \\ ${ }^{1}$ Universidade Federal do Rio Grande do Norte - Escola Agrícola de Jundiaí - Macaíba, RN \\ ${ }^{2}$ Universidade Federal de Minas Gerais - Escola de Veterinária - Belo Horizonte, MG
}

\begin{abstract}
RESUMO
Avaliou-se o efeito da densidade de estocagem na produção de "cachadia” (Pseudoplatystoma fasciatum x Leiarius marmoratus) em sistema de recirculação de água. Nas duas primeiras fases, com duração de 27 e 40 dias e usando animais com média de peso inicial de 31 e 57g, respectivamente, foram testados 20, 40, 60 e 80 peixes $/ \mathrm{m}^{3}$. Na terceira e quarta fase, usando peixes com média de peso de 169 e $399 \mathrm{~g}$ durante 41 e 99 dias, respectivamente, foram testados 10, 20, 30 e 40 peixes $/ \mathrm{m}^{3}$. A taxa de sobrevivência foi acima de $92 \%$, sem diferenças significativas entre tratamentos. Verificou-se redução no ganho de peso diário com redução com o incremento da densidade na segunda e na quarta fase experimental. A biomassa final apresentou relação direta com a densidade em todas as fases. Taxas de conversão alimentar entre 0,8 e 1,3:1 foram registradas. O "cachadia” atingiu 1,1kg em 207 dias de cultivo, mostrando-se promissor para a piscicultura intensiva.
\end{abstract}

Palavras-chave: peixe, Pseudoplatystoma fasciatum, Leiarius marmoratus, híbrido

\begin{abstract}
The aim of this study was to investigate the effect of different stocking densities on cachadia production (Pseudoplatystoma fasciatum $x$ Leiarius marmoratus) in recirculating aquaculture system. In the two first phases for 27 and 40 days using fish with average weight of 31 and 57g, respectively, densities 20, 40,60, and $80 \mathrm{fish} / \mathrm{m}^{3}$ were tested. In the third and fourth trial, using fish with an average weight of 169 and $399 \mathrm{~g}$ during 41 and 99 days, respectively, densities 10, 20, 30, and $40 \mathrm{fish} / \mathrm{m}^{3}$ were tested. Survival rate was higher than 92\%, without stocking density differences between the treatments. Daily weight gain showed reduction with the increase of the density in the second and fourth trial. However, final biomass presented direct relationship with increase density in all trials. Feed conversion rates ranged 0.8 from 1.3:1 were registered. Cachadia hybrid reached $1.1 \mathrm{~kg}$ in approximately seven months of rearing indicating promise for intensive production.
\end{abstract}

Keywords: fish, Pseudoplatystoma fasciatum, Leiarius marmoratus, hybrid

\section{INTRODUÇÃO}

O “cachadia” é um híbrido do cruzamento entre a fêmea do cachara (Pseudoplatystoma fasciatum) e o macho do jundiá do Norte (Leiarius marmoratus), espécies carnívora e onívora, respectivamente, o qual vem sendo produzido em algumas regiões do Brasil. Contudo, a literatura sobre este híbrido é escassa e, nos trabalhos consultados, não foram encontrados estudos de crescimento em condições intensivas de cultivo.

Recebido em 3 de maio de 2010

Aceito em 4 de julho de 2011

*Autor para correspondência (corresponding author)

E-mail: paulomcfaria@yahoo.com.br 
Dentre os sistemas intensivos de produção o uso de recirculação de água, em que biofiltros são utilizados com o objetivo de diminuir a concentração de amônia, por meio da sua oxidação a nitrato pelas bactérias nitrificantes (Hagopian e Riley, 1998), constitui uma das tendências mundiais. Esse fato se deve à limitação de água em qualidade e quantidade e visa diminuir a descarga residual que pode levar a impactos ambientais (Gutierrez-Wing e Malone, 2006). Nesse tipo de sistema, a densidade de estocagem é uma variável que vem sendo avaliada para diferentes espécies e em diferentes fases de cultivo (Faulk et al., 2007; Karakatsouli et al., 2007; Webb Jr. et al., 2007; van de Nieuwegiessen et al., 2008).

O emprego da densidade de estocagem adequada é benéfico comercialmente, pois a utilização do tanque, da água e dos recursos econômicos é maximizadas (Fairchild e Howell, 2001). Além disso, o uso de número reduzido de animais leva a uma subutilização do espaço disponível para a criação, enquanto o aumento da densidade pode ser prejudicial, principalmente pelos resíduos nitrogenados excretados pelos peixes, com prejuízos na qualidade da água e na sanidade (Jobling, 1994). Densidades inadequadas também podem afetar o desempenho dos peixes (Hecht e Uys, 1997; Papoutsoglou et al., 1998; Irwin et al., 1999), logo, em sistema de recirculação, onde existe a necessidade de maior investimento inicial em infraestrutura, equipamento e mão de obra especializada, a densidade deve ser avaliada criteriosamente na determinação dos custos e da viabilidade econômica.

O objetivo deste trabalho foi avaliar o efeito da densidade de estocagem na produção do híbrido "cachadia” (Pseudoplatystoma fasciatum x Leiarius marmoratus), em sistema de recirculação de água em diferentes fases de crescimento.

\section{MATERIAL E MÉTODOS}

O experimento foi realizado no Laboratório de Aquacultura da Universidade Federal de Minas Gerais, em Belo Horizonte, com duração de 207 dias. Foram consideradas quatro fases experimentais, cada uma realizada em delineamento inteiramente ao acaso, com quatro diferentes densidades de estocagem e quatro repetições cada.

Para a primeira fase experimental (fase 1 de crescimento), juvenis híbridos de "cachadia",

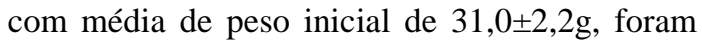
estocados nas densidades de 20, 40, 60 e 80 juvenis $/ \mathrm{m}^{3}$. Os peixes foram alimentados com dieta comercial extrusada de 2,0mm de diâmetro e $45 \%$ de proteína bruta. Esta fase teve duração de 27 dias. Devido ao pequeno tamanho dos péletes, durante esta fase não foi possível a coleta do alimento não consumido, razão pela qual não foi determinada a conversão alimentar.

Na segunda fase (fase 2 de crescimento), híbridos de "cachadia”, com média de peso $57,3 \pm 8,2 \mathrm{~g}$, foram estocados nas densidades de $20,40,60$ e 80 juvenis $/ \mathrm{m}^{3}$. Os peixes foram alimentados com dieta extrusada com 4,0mm de diâmetro e $40 \%$ de proteína bruta. Esta fase teve duração de 40 dias.

A terceira fase (fase 3 de crescimento) teve duração de 41 dias. Híbridos de "cachadia" com $169,3 \pm 6,1 \mathrm{~g}$ foram estocados nas densidades de $10,20,30$ e 40 juvenis $/ \mathrm{m}^{3}$. O alimento oferecido foi uma dieta extrusada com 4,0mm de diâmetro e $40 \%$ de proteína bruta.

Para a etapa final ou quarta fase experimental (fase 4 de crescimento ou fase final), peixes com média de peso de $399,8 \pm 5,0 \mathrm{~g}$ foram estocados nas densidades de 10, 20, 30 e 40 juvenis $/ \mathrm{m}^{3}$, durante 99 dias. A dieta fornecida foi uma ração extrusada com $8,0 \mathrm{~mm}$ de diâmetro e $40 \%$ de proteína bruta.

O experimento foi realizado em sistema de recirculação de água (SRA), constituído de 20 tanques circulares de 400L. Os tanques foram mantidos tampados para evitar a perda dos animais. No centro de cada tampa, foi feita uma abertura de $60 \mathrm{~cm}$ de diâmetro, fechada com tela de $1 \mathrm{~mm}$ entre nós. Por um sistema de escoamento comum, a água era direcionada para um filtro mecânico de brita. Posteriormente, a água era bombeada para um filtro pressurizado de areia (filtro tipo piscina) e, em seguida, para filtros biológicos. Após o tratamento, era enviada novamente para os tanques. A temperatura da água foi controlada em $28 \pm 1^{\circ} \mathrm{C}$ com o uso de aquecedores e termostato. $\mathrm{O}$ fotoperíodo foi mantido em 11 horas diárias. O oxigênio 
dissolvido (OD) manteve-se acima de $5,8 \pm 0,7 \mathrm{mg} / \mathrm{L}$ (medido com equipamento YSI) e o $\mathrm{pH}$ apresentou valores entre 6,4 e 7,1 (medidor portátil eletrônico Russel - RL06OP). O fluxo de água nos tanques foi de $480 \mathrm{l} / \mathrm{h}$ e aeração suplementar, por meio de pedra porosa, também foi instalada em cada tanque. Amônia total e nitrito foram medidos semanalmente usando-se kits comerciais (Kit Hach -DREL 2010) e apresentaram valores menores que $1,0 \mathrm{mg} / \mathrm{L}$ e $110 \mathrm{mg} / \mathrm{L}$, respectivamente.

Os peixes foram alimentados duas vezes ao dia, às sete e às 18 horas, com a dieta comercial extrusada até saciedade aparente. $\mathrm{O}$ alimento não consumido foi coletado com peneira de $0,5 \mathrm{~mm}$ de diâmetro 15 minutos após a alimentação. Esta ração foi seca a $55^{\circ} \mathrm{C}$ por $12 \mathrm{~h}$ e pesada para estimar o consumo de ração e a conversão alimentar. Os tanques foram limpos diariamente pela manhã, e $10 \%$ do volume total do SRA foram renovados.

Ao final de cada fase experimental, os animais foram agrupados, e peixes grandes e pequenos em relação à média foram excluídos. Posteriormente, foram redistribuídos nas densidades desejadas em cada fase experimental. Em quatro tanques (tanques de manutenção), juvenis foram mantidos nas mesmas condições descritas anteriormente (OD, $\mathrm{pH}$, temperatura, amônia, nitrito e alimentação). Animais que apresentassem o tamanho adequado foram usados para a reposição de indivíduos descartados, com o objetivo de manter a densidade desejada nas diferentes fases experimentais.

Os animais foram pesados individualmente ao início e ao final de cada fase experimental para o cálculo do ganho de peso diário, biomassa inicial e final. O consumo foi calculado pela diferença entre o alimento oferecido e as sobras, que foram coletadas, secas em estufas a $55^{\circ} \mathrm{C}$ por 48 horas e pesadas.

O desempenho foi avaliado pelos seguintes parâmetros: ganho de biomassa (g) = biomassa final - biomassa inicial; consumo de ração $(\mathrm{g})=$ (alimento oferecido - sobra)/número de peixes; consumo diário de ração $(\mathrm{g})=$ consumo de ração/dias de experimento; Ganho de peso (g) = peso final - peso inicial/número de peixes; ganho de peso diário $(\mathrm{g})$ = ganho de peso/dias de experimento; conversão alimentar = consumo de alimento/ganho de peso.

Para cada fase experimental, os resultados foram submetidos à análise de variância para se verificar se houve significância $(\mathrm{P}<0,05)$. Quando houve diferenças, foram determinados modelos de regressão que mais se aproximaram dos resultados. Para as análises estatísticas, utilizou-se o programa SAEG 8.0.

\section{RESULTADOS E DISCUSSÃO}

O “cachadia” mostrou boa adaptação e crescimento em sistema de recirculação de água nas densidades de estocagem avaliadas. As diferentes densidades não afetaram a sobrevivência, que foi acima de $92 \%$ na primeira fase experimental (Tab. 1) e de $100 \%$ nas demais fases (Tab. 2, 3 e 4). Em sistema de recirculação de água, diferentes densidades também não afetaram a sobrevivência de juvenis de cobia, Rachycentron canadum, com sobrevivência acima de 96\% (Webb Jr. et al., 2007). Sobrevivência semelhante às verificadas para o “cachadia”, entre 95 e 97\%, foi registrada para juvenis de Pseudoplatystoma spp., com média de peso de $50 \mathrm{~g}$, estocados nas densidades entre 35 e 105 peixes $/ \mathrm{m}^{3}$, após 105 dias em tanques-rede (Turra et al., 2009). Contudo, híbridos de $P$. coruscans x $P$. fasciatum, com média de peso inicial de $500 \mathrm{~g}$, estocados em tanques-rede, em densidades entre 50 e 125 peixes $/ \mathrm{m}^{3}$, apresentaram sobrevivência entre 81 e 91\%, valores mais baixos que no presente trabalho, mas, também, sem diferenças entre as densidades (Coelho e Cyrino, 2006). Para P. coruscans, com média de peso inicial de $72 \mathrm{~g}$, cultivados nas densidades de 67 e 133 peixes $/ \mathrm{m}^{3}$, a sobrevivência foi de 69,5 a 70,5\%, respectivamente (Scorvo Filho et al., 2008). Dessa forma, a densidade de estocagem não parece ser um fator limitante para a sobrevivência de "cachadia", de alguns híbridos e de outras espécies de comportamento semelhante ou não, em diferentes fases e condições de cultivo descritas acima. 
Tabela 1. Valores médios ( \pm desvio-padrão) de desempenho de híbrido de “cachadia” (Pseudoplatystoma fasciatum x Leiarius marmoratus) com peso médio inicial de $31 \mathrm{~g}$ após 27 dias de cultivo em diferentes densidades de estocagem (primeira fase experimental)

\begin{tabular}{lccccc} 
& \multicolumn{5}{c}{ Densidade $\left(\right.$ peixes $\left./ \mathrm{m}^{3}\right)$} \\
\cline { 2 - 6 } & 20 & 40 & 60 & 80 & $\mathrm{CV}$ \\
\hline Peso inicial (g) & $30,8 \pm 1,4$ & $31,3 \pm 1,5$ & $31,3 \pm 1,5$ & $30,8 \pm 0,5$ & 4,2 \\
Peso final (g) $^{\text {ns }}$ & $58,3 \pm 7,3$ & $56,7 \pm 6,9$ & $52,4 \pm 3,2$ & $58,3 \pm 3,9$ & 9,9 \\
Biomassa inicial (g) $_{\text {Biomassa final (g) }}^{1}$ & $246,8 \pm 11,3$ & $501,1 \pm 24,6$ & $751,2 \pm 36,8$ & $986,6 \pm 17,2$ & 3,9 \\
Ganho de biomassa (g) $^{2}$ & $453,1 \pm 77,7$ & $869,7 \pm 151,4$ & $1258,9 \pm 77,4$ & $1715,8 \pm 50.7$ & 9,0 \\
Ganho de peso diário (g) $^{\mathrm{ns}}$ & $206,9 \pm 70,5$ & $368,6 \pm 148,4$ & $507,6 \pm 92,4$ & $729,2 \pm 66,6$ & 22,0 \\
Sobrevivência (\%) $^{\text {ns }}$ & $1,0 \pm 0,2$ & $0,9 \pm 0,2$ & $0,7 \pm 0,1$ & $1,0 \pm 0,2$ & 21,8 \\
\hline
\end{tabular}

${ }^{1}$ Biomassa final $(\mathrm{g})=20,87 \mathrm{x}+30,72\left(\mathrm{R}^{2}=0,99\right)$.

${ }^{2}$ Ganho de biomassa $(\mathrm{g})=26,62 \mathrm{x}+85,30\left(\mathrm{R}^{2}=0,99\right)$.

${ }^{n s}$ Sem diferença significativa.

$\mathrm{x}=$ densidade de estocagem em peixes $\mathrm{m}^{-3}$.

Tabela 2. Valores médios ( \pm desvio-padrão) de desempenho de híbrido de “cachadia” (Pseudoplatystoma fasciatum x Leiarius marmoratus) com peso médio inicial de $57 \mathrm{~g}$ após 40 dias de cultivo em diferentes densidades de estocagem (segunda fase experimental)

\begin{tabular}{|c|c|c|c|c|c|}
\hline & \multicolumn{5}{|c|}{ Densidade (peixes $/ \mathrm{m}^{3}$ ) } \\
\hline & 20 & 40 & 60 & 80 & $\mathrm{CV}$ \\
\hline Peso inicial (g) & $58,8 \pm 6,6$ & $57,4 \pm 6,2$ & $54,2 \pm 3,4$ & $58,4 \pm 4,1$ & 9,2 \\
\hline Peso final $(g)^{1}$ & $230,9 \pm 13,5$ & $200,9 \pm 28,8$ & $177,7 \pm 15,2$ & $172,5 \pm 16,7$ & 10,0 \\
\hline Biomassa inicial (g) & $470,9 \pm 53,3$ & $919,9 \pm 99,7$ & $1302,2 \pm 82,6$ & $1870,1 \pm 130,3$ & 8,3 \\
\hline Biomassa final $(\mathrm{g})^{2}$ & $1.847,9 \pm 108,0$ & $3.214,8 \pm 462,1$ & $4.265,2 \pm 365,9$ & $5.521,7 \pm 536,7$ & 10,8 \\
\hline${\text { Ganho em biomassa }(\mathrm{g})^{3}}^{3}$ & $1.377,0 \pm 73,2$ & $2.294,8 \pm 373,6$ & $2.963,0 \pm 326,5$ & $3.651,5 \pm 417,7$ & 12,6 \\
\hline Ganho de peso diário (g) ${ }^{4}$ & $4,1 \pm 0,2$ & $3,4 \pm 0,6$ & $3,0 \pm 0,3$ & $2,7 \pm 0,32$ & 11,3 \\
\hline Consumo diário de ração $(\mathrm{g})^{5}$ & $4,1 \pm 1,1$ & $3,0 \pm 11.3$ & $2,7 \pm 11,2$ & $2,3 \pm 18,2$ & 17,2 \\
\hline Conversão alimentar ${ }^{\mathrm{ns}}$ & $1,0 \pm 0,1$ & $1,2 \pm 0,1$ & $1,1 \pm 0,1$ & $1,2 \pm 0,2$ & 11,9 \\
\hline Sobrevivência (\%) ${ }^{\mathrm{ns}}$ & 100 & 100 & 100 & 100 & - \\
\hline
\end{tabular}

${ }^{1}$ Peso final $(\mathrm{g})=-0,99 \mathrm{x}+245,17\left(\mathrm{R}^{2}=0,92\right)$.

${ }^{2}$ Biomassa final $(\mathrm{g})=60,35 \mathrm{x}+649,5\left(\mathrm{R}^{2}=0,99\right)$.

${ }^{3}$ Ganho em biomassa $=37,4 \mathrm{x}+698,6\left(\mathrm{R}^{2}=0,99\right)$.

${ }^{4}$ Ganho de peso diário $(\mathrm{g})=-0,023 \mathrm{x}+4,55,\left(\mathrm{R}^{2}=0,95\right)$.

${ }^{5}$ Consumo diário de ração $(\mathrm{g})=-0,018+4,79\left(\mathrm{R}^{2}=0,98\right)$.

${ }^{\text {ns }}$ Sem diferença significativa.

$\mathrm{x}=$ densidade de estocagem em peixes $\mathrm{m}^{-3}$.

Tabela 3. Valores médios ( \pm desvio-padrão) de desempenho de híbrido de "cachadia” (Pseudoplatystoma fasciatum x Leiarius marmoratus) com peso médio inicial de 169g após 41 dias de cultivo em diferentes densidades de estocagem (terceira fase experimental)

\begin{tabular}{|c|c|c|c|c|c|}
\hline & \multicolumn{5}{|c|}{ Densidade (peixes $/ \mathrm{m}^{3}$ ) } \\
\hline & 10 & 20 & 30 & 40 & $\mathrm{CV}$ \\
\hline Peso inicial (g) & $168,4 \pm 0,6$ & $166,7 \pm 1,2$ & $171,9 \pm 0,5$ & $170,0 \pm 4,8$ & 1,4 \\
\hline Peso final (g) ${ }^{\text {ns }}$ & $405,8 \pm 45,0$ & $405,6 \pm 29,6$ & $413,7 \pm 27,9$ & $376,1 \pm 12,0$ & 7,8 \\
\hline Biomassa inicial (g) & $1347,2 \pm 4,7$ & $2585,4 \pm 182,2$ & $4127,6 \pm 11,6$ & $5395,0 \pm 103,2$ & 3,1 \\
\hline Biomassa final $(\mathrm{g})^{1}$ & $3.246,7 \pm 367,5$ & $6.274,4 \pm 399,5$ & $9.929,3 \pm 670,7$ & $11.936,2 \pm 438,7$ & 6,1 \\
\hline Ganho em biomassa $(\mathrm{g})^{2}$ & $1.899,5 \pm 366,6$ & $3.689,0 \pm 373,3$ & $5.801,6 \pm 681,7$ & $6.541,1 \pm 337,5$ & 10,2 \\
\hline Ganho de peso diário (g) ${ }^{\text {ns }}$ & $5,8 \pm 1,1$ & $5,8 \pm 0,7$ & $5,9 \pm 0,7$ & $5,0 \pm 0,2$ & 13,4 \\
\hline Consumo diário de ração $(\mathrm{g})^{\mathrm{ns}}$ & $5,5 \pm 1,2$ & $5,6 \pm 0,7$ & $5,1 \pm 0,5$ & $4,2 \pm 0,3$ & 14,2 \\
\hline Conversão alimentar ${ }^{\text {ns }}$ & $0,9 \pm 0,1$ & $0,9 \pm 0,1$ & $0,8 \pm 0,03$ & $0,8 \pm 0,1$ & 6,9 \\
\hline Sobrevivência (\%) ${ }^{\text {ns }}$ & 100 & 100 & 100 & 100 & - \\
\hline
\end{tabular}

${ }^{1}$ Biomassa final $=297,23 x+415,87\left(R^{2}=0,99\right)$.

${ }^{2}$ Ganho em biomassa $=160,3 \mathrm{x}+473,47\left(\mathrm{R}^{2}=0,97\right)$.

${ }^{\text {ns }}$ Sem diferença significativa. $\mathrm{x}=$ número de peixes $\mathrm{m}^{-3}$. 
Tabela 4. Valores médios ( \pm desvio-padrão) de desempenho de híbrido de "cachadia” (Pseudoplatystoma fasciatum x Leiarius marmoratus) com peso médio inicial de 399g após 99 dias de cultivo em diferentes densidades de estocagem (quarta fase experimental)

\begin{tabular}{lccccc} 
& \multicolumn{5}{c}{ Densidade $\left(\right.$ peixes $\left./ \mathrm{m}^{3}\right)$} \\
\cline { 2 - 6 } & 10 & 20 & 30 & 40 & $\mathrm{CV}$ \\
\hline Peso inicial (g) & $399,5 \pm 3,7$ & $400,4 \pm 1,0$ & $399,8 \pm 2,9$ & $399,5 \pm 2,1$ & 0,6 \\
Peso final (g) & $1.287,1 \pm 104,8$ & $1.159,4 \pm 38,7$ & $1.049,9 \pm 46,6$ & $901,2 \pm 25,5$ & 5,6 \\
Biomassa Inicial & $1.598,0 \pm 14,8$ & $3.203,3 \pm 7,7$ & $4.798,7 \pm 35,0$ & $6.393,4 \pm 34,2$ & 0,6 \\
Biomassa final (g) & $5.148,5 \pm 419,2$ & $9.275,8 \pm 309,7$ & $12.599,9 \pm 559,1$ & $14.419,5 \pm 405,6$ & 4,1 \\
Ganho em biomassa (g) $^{3}$ & $3.550,4 \pm 431,9$ & $6.072,5 \pm 312,5$ & $7.801,2 \pm 530,9$ & $8.026,1 \pm 403,0$ & 6,7 \\
Ganho de peso diário (g) $^{4}$ & $8,9 \pm 1,1$ & $7,6 \pm 0,4$ & $6,5 \pm 0,4$ & $5,0 \pm 0,3$ & 8,9 \\
Consumo diário de ração (g) $^{5}$ & $12,3 \pm 1,2$ & $8,5 \pm 0,3$ & $7,2 \pm 0,7$ & $6,4 \pm 0,7$ & 9,2 \\
Conversão alimentar $^{6}$ & $1,3 \pm 0,1$ & $1,1 \pm 0,0$ & $1,0 \pm 0,1$ & $1,2 \pm 0,1$ & 6,9 \\
Sobrevivência (\%) $^{\text {ss }}$ & 100 & 100 & 100 & 100 & - \\
\hline
\end{tabular}

${ }^{1}$ Peso final $=-12,67 \mathrm{x}+1416,2\left(\mathrm{R}^{2}=0,99\right)$.

${ }^{2}$ Biomassa final $=311,37 \mathrm{x}+2576,6\left(\mathrm{R}^{2}=0,97\right)$.

${ }^{3}$ Ganho em biomassa $=-5,74 x^{2}+438,69 x-297,73\left(R^{2}=0,99\right)$.

${ }^{4}$ Ganho de peso diário $=-0,12 \mathrm{x}+10,26\left(\mathrm{R}^{2}=0,99\right)$.

${ }^{5}$ Consumo diário de ração $=0,00757 \mathrm{x}^{2}-0,0571 \mathrm{x}+17,22\left(\mathrm{R}^{2}=0,99\right)$.

${ }^{6}$ Conversão alimentar $=0,00109 x^{2}-0,058 x+1,85\left(R^{2}=0,99\right)$.

${ }^{\text {ns }}$ Sem diferença significativa.

$\mathrm{x}=$ número de peixes $\mathrm{m}^{-3}$.

O peso final e o ganho de peso diário não foram influenciados pela densidade na primeira fase experimental (Tab. 1). Contudo, sofreram efeito negativo da densidade de estocagem na segunda fase, com consequente redução no consumo diário de ração com o aumento da densidade (Tab. 2). Este fato indica que, para peixes maiores, o aumento da densidade na segunda fase produz peixes de menor peso final em função do menor consumo de ração. Na terceira fase, peso final, ganho de peso diário e consumo diário de ração não foram influenciados pela densidade (Tab. 3). Na quarta fase, utilizando a mesma densidade da terceira, porém com animais maiores, houve relação inversamente proporcional para peso final e ganho de peso, enquanto o consumo diário de ração apresentou efeito quadrático (Tab. 4). Este fato corrobora o registrado entre a primeira e a segunda fase experimental. Dessa forma, os resultados apresentados sugerem relação entre o tamanho dos indivíduos e a densidade a ser empregada pelo fato de proporcionarem mais biomassa por volume estocado.

Apesar da redução do peso final nas fases 2 e 4 com o incremento da densidade, a sobrevivência semelhante entre os tratamentos contribuiu para o incremento de biomassa diretamente proporcional com o aumento da densidade nas quatro fases experimentais (Tab. 1, 2, 3 e 4). O incremento da biomassa com o aumento da densidade também tem sido registrado para várias espécies em diferentes fases de vida e sistemas de cultivo (Terhune et al., 1992; Graeff, 2004; Coelho e Cyrino, 2006; Turra et al., 2009). Este fato é refletido também no ganho em biomassa nas fases 1,2 e 3 , que foram diretamente proporcionais ao incremento da densidade. Contudo, na quarta fase, verificou-se efeito quadrático, pois a densidade ideal estimada seria de 38 peixes $/ \mathrm{m}^{3}$ (Tab. 4).

Dessa forma, esses dados devem ser levados em consideração na análise econômica para avaliar se é viável ou não o cultivo deste híbrido em sistema de recirculação de água. Para híbridos de Pseudoplatystoma, cultivados em tanques-rede, maiores densidades apresentam maiores custos variáveis, principalmente pelo preço dos juvenis e da ração (Coelho e Cyrino, 2006). Porém, ainda nesse estudo, os autores verificaram que, na densidade mais elevada, a maior biomassa final produzida levou à melhor receita líquida. Fato semelhante também foi verificado na análise econômica do cultivo de Cyprinus carpio em diferentes densidades (Graeff, 2004). Segundo o autor, a lucratividade da atividade é crescente com o aumento da densidade, com diminuição do custo médio ou unitário. 
Durante a segunda e a terceira fase experimental, a conversão alimentar não foi afetada pelas diferentes densidades testadas (Tab. 2 e 3). Na quarta fase, houve efeito quadrático (Tab. 4). Pela derivação da equação, a densidade de 25 peixes $/ \mathrm{m}^{3}$ levaria à melhor conversão alimentar. Contudo, as taxas de conversão alimentar registradas durante o experimento variaram de 0,8 a 1,3:1. Turra et al. (2009) registraram para Pseudoplatystoma spp., cultivados em tanquesrede com peso médio inicial de $50 \mathrm{~g}$, taxas de conversão alimentar de 1,4,1,6, e 1,5:1 para as densidades de 35, 70 e 105 indivíduos $/ \mathrm{m}^{3}$, respectivamente, também sem diferenças significativas entre as densidades. Juvenis de Hoplias lacerdae, com peso médio inicial de 12,5g, apresentaram conversão alimentar de 1,6 e 1,2 para as densidades de 1 e 4 peixes $/ \mathrm{m}^{2}$, respectivamente (Salaro et al., 2003). Os resultados deste trabalho foram melhores que os verificados para híbridos de Pseudoplatystoma, que apresentaram taxa de conversão alimentar maior que 3,7:1 em tanques-rede, estocados em diferentes densidades (Coelho e Cyrino, 2006). Kubitza et al. (1998) mostraram variação nos valores de conversão alimentar em relação ao peso dos peixes. Esses valores variaram de 1,3:1 para indivíduos de 5 a 50g; 1,5:1 para os de 50 a $600 \mathrm{~g}$, e de 2,0:1 para peixes de 600 a 3000g. Apesar das diferenças de tamanho de "cachadia" ao longo do experimento, as taxas de conversão alimentar foram melhores que as relatadas por Kubtiza et al. (1998). Este fato, junto aos já discutidos anteriormente, reforça o potencial do "cachadia” para a piscicultura em sistema de recirculação de água.

O "cachadia" atingiu, em média, 1,1kg de peso vivo em sete meses de cultivo em sistema de recirculação de água. Estes resultados indicam o seu potencial para a aquacultura.

\section{CONCLUSÕES}

A produção do "cachadia” mostrou-se promissora para a piscicultura, uma vez que o desempenho produtivo foi bastante favorável. O sistema de recirculação de água utilizado neste sistema foi fundamental para este desempenho, uma vez que favoreceu boa qualidade da água e qualidade sanitária dos peixes, proporcionando maior bem estar animal durante todo o período experimental. Além de não lançar efluentes aos corpos hídricos, o sistema de recirculação de água torna-se uma boa alternativa para a produção de peixes híbridos mais produtivos, com menor risco de escape de animais geneticamente modificados para a natureza.

\section{AGRADECIMENTOS}

Agradecimentos ao CNPq, à CAPES e ao MPA$\mathrm{PR}$, pelo apoio financeiro.

\section{REFERÊNCIAS BIBLIOGRÁFICAS}

COELHO, S.R.C.; CYRINO, J.E.P. Custos na produção intensiva de surubins em gaiolas. Inf. Econ., v.36, p.7-14, 2006.

FAIRCHILD, E.A.; HOWELL, W.H. Optimal stockimg density for juvenile winter flounder Pseudopleuronectes americanus. J. World Aquacult. Soc., v.32, p.300-308, 2001.

FAULK, C.K.; KAISER, J.B.; HOLT, G.J. Growth and survival of larval and juvenile cobia Rachycentron canadum in a recirculating raceway system. Aquaculture, v.270, p.149-157, 2007.

GRAEFF, A. Viabilidade econômica do cultivo de carpa comum (Cyprinus carpio Linnaeus, 1758) em monocultivo em densidades diferentes. Cienc. Agrotec., v.28, p.678-684, 2004.

GUTIERREZ-WING, M.T.; MALONE, R.F. Biological filters in aquaculture: trends and research directions for freshwater and marine applications. Aquacult. Eng., v.34, p.163-171, 2006.

HAGOPIAN, D.S.; RILEY J.G. A closer look at the bacteriology of nitrification. Aquacult. Eng., v.18, p.223-244, 1998.

HECTH, T.; UYS, W. Effect of density on the feeding and agressive behaviour in juvenile African catfish (Clarias gariepinus). S. Afr. J. Sci., v.93, p.537-541, 1997.

IRWIN, S.; O'HALLORAN, J.; FITZGERALD, R.D. Stocking density, growth variation in juvenile turbot, Scophthalmus maximus (Rafinesque). Aquaculture, v.178, p.77-88, 1999.

JOBLING, M. Fish bioenergetics. London: Chapman \& Hall, 1994. 294p.

KARAKATSOULI, N.; PAPOUTSOGLOU, S.E.; MANOLESSOS, G. Combined effects of rearing density and tank colour on the growth 
and welfare of juvenile white sea bream Diplodus sargus L. in a recirculating water system. Aquacult. Res., v.38, p.1152- 1160, 2007.

KUBITZA, F.; CAMPOS, J.L.; BRUM, J.A. Produção intensiva no projeto Pacu Ltda. Pan. Aquic., v.8, p.41-49, 1998.

PAPOUTSOGLOU, S.E.; TZIHA, G.; VRETTOS, X. et al. Effects of stocking density on behavior and growth rate of European sea bass (Dicentrarchus labrax) juveniles reared in a closed circulated system. Aquacult. Eng., v.18, p.135-144, 1998.

SALARO, A.L.; LUZ, R.K.; NOGUEIRA, G.C.C.B. et al. Diferentes densidades de estocagem na produção de alevinos de trairão (Hoplias cf. lacerdae). Rev. Bras. Zootec., v.32, p.1033-1036, 2003

SCORVO FILHO, J.D.; ROMAGOSA, E.; AYROZA, L.M.S. et al. Desempenho produtivo do pintado, Pseudoplatystoma corruscans (Spix \& Agassiz, 1829), submetido a diferentes densidades de estocagem em dois sistemas de criação: intensivo e semi-intensivo. Bol. Inst. Pesca, v.34, p.181-188, 2008.
TERHUNE, J.S.; TOMASSO, J.R.; SCHWEDLER, T.E. et al. Increasing yields of channel catfish using a combination of cage and open pond production system. J. World Aquacult. Soc., v.23, p.77-82, 1992.

TURRA, E.M.; QUEIROZ, B.M.; TEIXEIRA, E.A. et al. Densidade de estocagem do surubim Pseudoplatystoma spp. cultivado em tanquerede. Rev. Bras. Saúde Prod. Anim., v.10, p.177187, 2009.

Van de NIEUWEGIESSEN, P.G.; BOERLAGE, A.S.; VERRETH, J.A.J. et al. Assessing the effects of a chronic stressor, stocking density, on welfare indicators of juvenile African catfish, Clarias gariepinus Burchell. Appl. Anim. Behav. Sci., v.115, p.233-243, 2008.

WEBB Jr., K.A.; HITZFELDER, G.M.; FAULK, C.K. et al. Growth of juvenile cobia, Rachycentron canadum, at three different densities in a recirculating aquaculture system. Aquaculture, v.264, p.223-227, 2007. 